\title{
Potential and challenges of biominerals for applications in construction and mining industry
}

\author{
SAKSHI TIWARI, ASHA L RAMACHANDRAN, ABHIJIT \\ MUKHERJEE AND NAVDEEP K DHAMI
}

\section{Curtin University}

Presenting Author: navdeep.dhami@curtin.edu.au

Biogeochemical activities leading to precipitation of carbonates, phosphates, sulfates, iron oxides/hydroxides minerals have been illustrated in a variety of natural geological formations including microbialites, corals, beach rocks, whiting events. Microbial physiological activities are known to have a significant impact on the mineral reaction kinetics, precipitation as well as microstructural properties of these biominerals. Mimicking these natural biogeochemical processes, a range of biominerals have been created under laboratory conditions including carbonates, phosphates, iron oxides/hydroxides and promising applications of these biominerals have been demonstrated in different sectors of environment, construction and recently in the mining industry. This includes applications in metal remediation, soil reinforcement, construction restoration, crack sealing, dust stabilisation, in-situ barriers, solidification of mine tailings and slope stabilisation.

We have investigated the potential of a variety of microbial cultures, communities, biofilms and exopolymeric substances in creation of different biominerals and studied the impact of microbial metabolic routes, physicochemical conditions, underlying substrate properties on biofilm adhesion, precipitation kinetics and morpho-mineralogical properties of carbonate biominerals. For analysis of morpho-mineralo-nanomechanical properties of carbonate biominerals, we have utilised a range of techniques including X-ray diffraction (XRD), Tescan integrated mineral analyser (TIMA), energy dispersive X-ray spectroscopy (EDS), Fourier transform infrared microscopy (FT-IR), atomic force microscopy (AFM), scanning electron microscopy (SEM), transmission electron microscopy (TEM) and nanoindentation. We have found that microbial metabolic route, mode of enrichment (biostimulation or bioaugmentation) and physicochemical conditions play a significant role in determining the physical properties of biominerals; which eventually plays a significant role in enabling their engineering applications. We have reviewed the impact of microbial-mineral interactions, organic-inorganic components, community structures and metabolic routes on properties of other biominerals (phosphates and iron based biominerals) used widely for mining and construction applications; an important step towards the rational design of these bacterially based engineering solutions. Current limitations and challenges of biominerals along with future direction of research have been discussed. Our studies shed light on the breadth of biomineralisation activity amongst environmental bacteria in different physical conditions but highlights the importance of selecting the specific metabolic route and physicochemical conditions to tailor the material properties of biogenic minerals for specific engineering applications. 